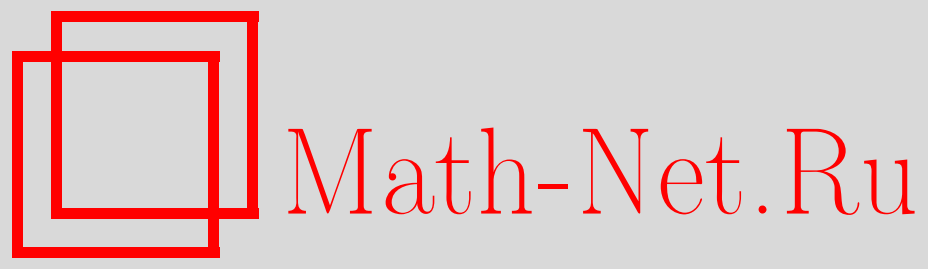

М. И. Островский, В. А. Хацкевич, В. С. Шульман, Квадратные операторные неравенства и дробнолинейные отношения, Функи. анализ и его прил., 2007, том 41, выпуск 4, 83-87

DOI: https://doi.org/10.4213/faa2884

Использование Общероссийского математического портала MathNet.Ru подразумевает, что вы прочитали и согласны с пользовательским соглашением

http://www . mathnet.ru/rus/agreement

Параметры загрузки:

IP : 54.174 .149 .18

26 апреля 2023 г., 16:15:42

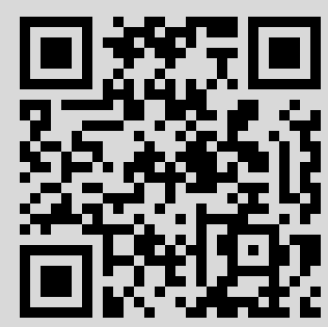


УДК 917.983.248

\section{Квадратные операторные неравенства и дробно-линейные отношения}

(c) 2007. М. И. ОСТРовСКИЙ, В. А. ХАЦКЕВИЧ, В. С. ШУЛЬМАН

1. Введение. Пусть $\mathscr{H}_{1}$ и $\mathscr{H}_{2}$ - гильбертовы пространства, $B \in \mathscr{L}\left(\mathscr{H}_{1}, \mathscr{H}_{2}\right)$, $A \in \mathscr{L}\left(\mathscr{H}_{2}\right), A=A^{*}, C \in \mathscr{L}\left(\mathscr{H}_{1}\right), C=C^{*}$.

Общеизвестная и часто используемая (простая в случае гильбертовых пространств) теория линейных операторных уравнений вида $A X=B$ или $X C=B$ описывает свойства пространств решений $X \in \mathscr{L}\left(\mathscr{H}_{1}, \mathscr{H}_{2}\right)$ этих уравнений и, в частности, дает критерии нетривиальности этих пространств (условия разрешимости). В то же время, во многих разделах функционального анализа (расширения операторов [8], пространства с индефинитной метрикой [6], дифференциальные уравнения в гильбертовых пространствах [5], операторные функциональные уравнения [3], теория контроля [7] и др.) приходится рассматривать квадратные уравнения и неравенства вида

$$
\begin{aligned}
& X^{*} A X=C, \\
& X^{*} A X \leqslant C,
\end{aligned}
$$

а также более общие неравенства

$$
X^{*} A X+B^{*} X+X^{*} B+C \leqslant 0 .
$$

Мы будем обозначать через $E(A, C), N(A, C)$ и $M(A, B, C)$ множества всех решений уравнения (1) и неравенств (2) и (3) соответственно. Нас будут интересовать основные свойства этих множеств и, в частности, условия, при которых они нетривиальны, замкнуты в стандартных операторных топологиях, имеют непустую внутренность, являются выпуклыми. Несмотря на простоту и естественность формулировок, эти вопросы нельзя назвать хорошо изученными. Одна из целей данной работы - привлечь внимание специалистов по теории операторов к этой проблематике.

Наш собственный интерес к рассматриваемым задачам возник в процессе работы над вопросами теории дробно-линейных операторных отношений (ДЛО), или, иначе, «многозначных дробно-линейных отображений». Напомним определения.

Пусть $\mathscr{H}_{i}, \mathscr{H}_{i}^{\prime}, i=1,2$, - гильбертовы пространства, а $T=\left(T_{i j}\right)_{i=1,2}$ операторная матрица, такая, что $\left(T_{i j}\right)$ действует из $\mathscr{H}_{j}$ в $\mathscr{H}_{i}^{\prime}$. Дробно-линейным отношением, определяемым матрицей $T$, называется отображение $R_{T}$ из $\mathscr{L}\left(\mathscr{H}_{1}, \mathscr{H}_{2}\right)$ в множество аффинных подпространств в $\mathscr{L}\left(\mathscr{H}_{1}^{\prime}, \mathscr{H}_{2}^{\prime}\right)$, заданное формулой

$$
R_{T}(K)=\left\{K^{\prime} \in \mathscr{L}\left(\mathscr{H}_{1}^{\prime}, \mathscr{H}_{2}^{\prime}\right): K^{\prime}\left(T_{11}+T_{12} K\right)=T_{21}+T_{22} K\right\} .
$$

Множество $\left\{K: R_{T}(K) \neq \varnothing\right\}$ называется областью определения ДЛО $R_{T}$ и обозначается через $\operatorname{dom} R_{T}$. 
Ограничение отношения $R_{T}$ на множество всех операторов из $\operatorname{dom} R_{T}$, для которых оператор $T_{11}+T_{12} K$ обратим, называется дробно-линейным операторным преобразованием (ДЛП); мы используем для его обозначения символ $Q_{T}$.

Нетрудно видеть, что многие естественные вопросы относительно свойств ДЛО $R_{T}$ : описание внутренних точек его области определения, его ограниченность (см. определение ниже) на некоторых специальных подмножествах и другие (в случае ДЛП изучавшиеся в многочисленных работах, см. [1] и цитируемую там литературу) - приводят к неравенствам вида (3). Результаты настоящей работы позволяют усилить и упростить некоторые результаты работы [4], в которой было начато систематическое изучение дробно-линейных отношений. В качестве одного из приложений мы доказываем аналог теоремы Лиувилля (ДЛО, ограниченное на своей области определения, постоянно). Мы изучаем также возможность перенесения этого результата на случай банаховых пространств.

2. Обозначения. Мы рассматриваем

$$
G(X)=X^{*} A X+B^{*} X+X^{*} B+C
$$

как функцию оператора $X \in \mathscr{L}\left(\mathscr{H}_{1}, \mathscr{H}_{2}\right)$. В этих терминах

$$
M(A, B, C)=\{X: G(X) \leqslant 0\}
$$

(и, разумеется, $N(A, C)=M(A, 0,-C)$ ).

Пусть $E^{C}-$ спектральная мера оператора $C$. Положим $\mathscr{H}_{1+}=E^{C}(0, \infty) \mathscr{H}_{1}$, $\mathscr{H}_{10}=E^{C}(\{0\}) \mathscr{H}_{1}, \mathscr{H}_{1-}=E^{C}(-\infty, 0) \mathscr{H}_{1} ;$ тогда $\mathscr{H}_{1}=\mathscr{H}_{1+} \oplus \mathscr{H}_{10} \oplus \mathscr{H}_{1-}$. Пусть $\mathscr{H}_{2}=\mathscr{H}_{2+} \oplus \mathscr{H}_{20} \oplus \mathscr{H}_{2-}$ - аналогичное разложение пространства $\mathscr{H}_{2}$, построенное по оператору $A$. Обозначим через $C_{+}, C_{0}$ и $C_{-}$ограничения оператора $C$ на подпространства $\mathscr{H}_{1+}, \mathscr{H}_{10}$ и $\mathscr{H}_{1-}$, а через $A_{+}, A_{0}, A_{-}$- ограничения оператора $A$ на $\mathscr{H}_{2+}, \mathscr{H}_{20}$ и $\mathscr{H}_{2-}$.

Если $\mathscr{H}_{1-}=0\left(\mathscr{H}_{1+}=0\right)$, то мы говорим, что оператор $C$ неотрицателен (неположителен). Далее, $C$ неотрицателен в существенном, если $C_{-}$компактен (это эквивалентно тому, что $C$ является суммой неотрицательного и компактного операторов).

3. Нетривиальность. Здесь мы обсудим условия, при которых неравенство (3) не имеет решений либо, напротив, при которых все операторы удовлетворяют этому неравенству. Последний вопрос рассматривался многими математиками, и следующий результат можно считать относящимся к математическому фольклору.

Предложение 1. Равенство $M(A, B, C)=\mathscr{L}\left(\mathscr{H}_{1}, \mathscr{H}_{2}\right)$ имеет место тогда и только тогда, когда

$$
\left(\begin{array}{cc}
A & B \\
B^{*} & C
\end{array}\right) \leqslant 0
$$

Займемся первым вопросом. Рассмотрим следующие (попарно несовместные) условия:

(i) $\operatorname{dim} \mathscr{H}_{1+}<\operatorname{dim} \mathscr{H}_{2-}$;

(ii) $\operatorname{dim} \mathscr{H}_{1+}=\operatorname{dim} \mathscr{H}_{2-}<\infty$;

(iii) $\operatorname{dim} \mathscr{H}_{1+}=\operatorname{dim} \mathscr{H}_{2-}=\infty$ и $A_{-}$не компактен; 
(iv) $\operatorname{dim} \mathscr{H}_{1+}=\operatorname{dim} \mathscr{H}_{2-}=\infty$, операторы $A_{-}$и $C_{+}$компактны, и модули их собственных значений, расположенные по убыванию, удовлетворяют неравенствам $\sup _{1 \leqslant i<\infty} c_{i} / a_{i}<\infty$.

Предложение 2. Множество $N(A,-C)$ непусто тогда и только тогда, когда выполнено одно из условий (i)-(iv).

Замечание 3. В некоторых случаях изучение структуры множества $M(A, B$, $C)$ сводится к изучению множества $N\left(A, C^{\prime}\right)$ для подходящего $C^{\prime}$. Это имеет место, в частности, когда образ оператора $B$ содержится в образе оператора $A$. Действительно, в этом случае $B=A D$ для некоторого $D \in \mathscr{L}\left(\mathscr{H}_{1}, \mathscr{H}_{2}\right)$ и

$$
G(X)=X^{*} A X+D^{*} A X+X^{*} A D+C=(X+D)^{*} A(X+D)+\left(C-D^{*} A D\right) .
$$

Следовательно,

$$
M(A, B, C)=N\left(A, D^{*} A D-C\right)-D
$$

Если это условие выполнено, то предложение 2 дает критерий непустоты множества $M(A, B, C)$ : достаточно применить его к операторам $A$ и $C^{\prime}$, где $C^{\prime}=D^{*} A D-C$.

\section{4. Топологические свойства.}

Теорема 4. (i) Множество $M(A, B, C)$ замкнуто в силъной операторной топологии.

(ii) Множество $M(A, B, C)$ тогда и только тогда является секвенииально замкнутым в слабой операторной топологии, когда либо $А$ неотрицателен в существенном, либо выполнено условие (6) (что эквивалентно совпадению множсеств $M(A, B, C)$ с $\left.\mathscr{L}\left(\mathscr{H}_{1}, \mathscr{H}_{2}\right)\right)$.

(iii) Если $A_{-}$- оператор конечного ранга, то $M(A, B, C)$ замкнуто в слабой операторной топологии. Обратное верно при условии, что образ оператора $B$ содержстся в образе оператора $A$.

Замечание 5. (i) Из теоремы 4 следует, что множества $N(A, C)$ и $E(A, C)=$ $N(A, C) \cap N(-A,-C)$ всегда замкнуты в сильной операторной топологии и что для замкнутости множества $N(A, C)$ в слабой операторной топологии необходимо (по модулю совпадения $N(A, C)$ со всем пространством операторов) и достаточно, чтобы $A_{-}$имел конечный ранг.

(ii) Пункт (i) теоремы можно усилить следующим образом: $M(A, B, C)$ является операторно рефлексивным. Последнее означает (см. [2]), что $M(A, B, C)$ содержит любой оператор $T$, удовлетворяющий условию $T x \in \overline{M(A, B, C) x}$ для всех $x \in \mathscr{H}_{1}$.

Следующий результат играет основную роль в доказательстве п. (iii) теоремы 4. Мы приводим его, надеясь, что он может представлять самостоятельный интерес.

Лемма 6. Если $A$ - неотрицательный оператор бесконечного ранга, то существует сеть операторов $E_{\lambda}$, стремящаяся $\kappa$ нулю в слабой операторной топологии и такая, что $E_{\lambda}^{*} A E_{\lambda} \geqslant A$ для всех $\lambda$.

Рассмотрим теперь вопрос о характеризации внутренних (относительно равномерной топологии) точек множества $M(A, B, C)$. Так как точки из $M(A, B, C)$ 
можно «сдвигать», меняя коэффициенты в (3), можно ограничиться рассмотрением условий, при которых 0 является внутренней точкой.

Для любого $r>0$ обозначим через $K(r)$ конус всех векторов $x \oplus y \in \mathscr{H}_{1} \oplus \mathscr{H}_{2}$, таких, что $\|x\| \leqslant r\|y\|$.

Предложение 7. Точка 0 является внутренней для $M(A, B, C)$ тогда $u$ только тогда, когда матрица

$$
\left(\begin{array}{cc}
A & B \\
B^{*} & C
\end{array}\right)
$$

неположительна на каком-то конусе $K(r)$.

5. Выпуклость. Известно (см., например, [5]), что если $A \geqslant 0$, то множество $M(A, B, C)$ выпукло. Кажется весьма вероятным, что верно и обратное, но мы смогли это доказать лишь при некоторых дополнительных ограничениях.

Теорема 8. Пусть $M(A, B, C)$ выпукло и нетривиально (m.е. непусто $u$ не совпадает с $\left.\mathscr{L}\left(\mathscr{H}_{1}, \mathscr{H}_{2}\right)\right)$. Если $\sigma(A)$ не пересекает некоторый интервал $(-\varepsilon, 0)$, mo $A \geqslant 0$.

Следствие 9. Множество $N(A, C)$ является выпуклым тогда и толъко тогда, когда либо оно пусто, либо совпадает с $\mathscr{L}\left(\mathscr{H}_{1}, \mathscr{H}_{2}\right)$, либо $A \geqslant 0$.

6. Теорема Лиувилля. Одним из следствий предложения 1 является тот факт, что если ДЛО $R_{T}$ определено и ограничено на всем пространстве операторов, то оно постоянно. Поскольку речь идет о многозначных отображениях, терминология нуждается в уточнении. Мы называем многозначное отображение $F$ постоянным на подмножестве $E$ области определения, если существует элемент, принадлежащий образам всех точек из $E$. Далее, $F$ называется ограниченным на $E$, если существует ограниченное множество $W$, пересекающееся с образами всех точек из $E$.

Будем рассматривать задачу в большей общности (для отображений банаховых пространств) и в более сильном варианте (отображение не предполагается определенным и ограниченным на всем пространстве). Оказывается, что ответ зависит от геометрии соответствующих банаховых пространств.

Мы рассматриваем и случай (однозначных) ДЛП. Напомним, что так называется отображение, определяемое формулой $Q_{T}(K)=\left(T_{21}+T_{22} K\right)\left(T_{11}+T_{12} K\right)^{-1}$, и его область определения состоит из всех операторов $K$, для которых оператор $T_{11}+T_{12} K$ обратим.

Пусть даны две пары банаховых пространств $\left(\mathscr{B}_{1}, \mathscr{B}_{2}\right),\left(\mathscr{B}_{1}^{\prime}, \mathscr{B}_{2}^{\prime}\right)$; отображения действуют из $\mathscr{L}\left(\mathscr{B}_{1}, \mathscr{B}_{2}\right)$ в $\mathscr{L}\left(\mathscr{B}_{1}^{\prime}, \mathscr{B}_{2}^{\prime}\right)$.

Теорема 10. (i) Пусть ДЛО $R_{T}$ ограничено на своей области определения. Если $\mathscr{B}_{2}^{\prime}$ рефлексивно (или, более общим образом, его образ при каноническом вложении во второе сопряженное дополняем), то $R_{T}$ постоянно.

(ii) Существуют банаховы пространства и ДЛО, которое определено и ограничено на всем пространстве операторов, но не является постоянным.

(iii) Если ДЛП $Q_{T}$ ограничено на своей области определения, то оно постоянно.

Авторы выражают искреннюю признательность рецензентам за полезные комментарии и предложения. 


\section{ЛитерАТУРА}

[1] Т. Я. Азизов, И. С. Иохвидов, Линейные преобразования в пространствах $с$ индебинитной метрикой, Наука, М., 1986. [2] J. B. Conway, A Course in Operator Theory, Grauate Studies in Math., vol. 21, Amer. Math. Soc., Providence, R.I., 2000. [3] C. C. Cowen, Trans. Amer. Math. Soc., 265:1 (1981), 69-95. [4] V. Khatskevich, M. I. Ostrovskii, V. Shulman, Math. Nachr., 279 (2006), 875-890. [5] V. Khatskevich, V. Shulman, Studia Math., 116 (1995), 189-195. [6] М. Г. Крейн, Ю. Л. Шмульян, Матем. исслед. (Кишинев), 2:3 (1967), 64-96. [7] Г. А. Курина, Изв. РАН, Техн. кибернетика, 1993, № 6, 33-38. [8] M. M. Malamud, in: Recent Advances in Operator Theory (Groningen, 1998), Oper. Theory Adv. Appl., vol. 124, Birkhäuser, Basel, 2001, 401-449.

Department of Math. and Comp. Sci.

Поступило в редакцию

St.-John's University, USA

20 октября 2005 г.

e-mail: ostrovsm@stjohns.edu

Department of Math.

ORT Braude College, Israel

e-mail: victor_kh@hotmail.com

Кафедра высшей математики

Вологодского государственного технического университета

e-mail: shulman_v@yahoo.com

УДК $517.951+517.954$

\section{Эйлерова характеристика фредгольмова квазикомплекса}

\section{(c) 2007. H. H. TAPXAHOB}

1. Введение. В категории гильбертовых пространств комплекс $(L, d)$ задается как последовательность гильбертовых пространств $L^{i}, i \in \mathbb{Z}$, вместе с непрерывными отображениями $d^{i}: L^{i} \rightarrow L^{i+1}$, такими, что $d^{i} \circ d^{i-1}=0$.

Мы будем писать просто $L$, если это не приводит к недоразумениям. Рассматривая ограниченные (конечные) комплексы, мы предполагаем, что $L^{i}=0$ для $i$, отличных от $0,1, \ldots, N$, в противном случае сдвигаем индексацию.

Пусть $Z^{i}(L)$ и $B^{i}(L)$ обозначают соответственно пространства коциклов и кограниц комплекса $L$ в степени $i$. Очевидно, что $B^{i}(L)$ является векторным подпространством в $Z^{i}(L)$. Говорят, что комплекс $L$ фредгольмов, если его когомологии $H^{i}(L)=Z^{i}(L) / B^{i}(L)$ конечномерны в каждой степени $i$. Для всякого фредгольмова комплекса $L$ эйлерова характеристика $\chi(L)$ определяется следующим соотношением:

$$
\chi(L)=\sum_{i=0}^{N}(-1)^{i} \operatorname{dim} H^{i}(L) .
$$

Предположим, что $d+V$ есть возмущение дифференциала $d$ с достаточно «малым» $V$. Под «малым» мы понимаем принадлежность подходящему идеалу компактных операторов. Тогда квадрат $(d+V)^{2}=d V+V d+V^{2}$ не равен нулю, 